\title{
Hydraulic permeability of ordered and disordered single-layer arrays of cylinders
}

\author{
M. P. Sobera* and C. R. Kleijn ${ }^{\dagger}$ \\ Department of Multi-Scale Physics, Delft University of Technology, Prins Bernhardlaan 6, 2628BW Delft, The Netherlands
}

(Received 26 April 2005; revised manuscript received 27 February 2006; published 13 September 2006)

\begin{abstract}
The hydraulic permeability of single-layer fibrous media is studied through two-dimensional (2D) and three-dimensional Navier-Stokes based flow simulations. As simple representations of such materials, onedimensional arrays of parallel cylinders as well as two-dimensional arrays of perpendicularly crossing cylinders were used. The distance between the cylinders was either constant (ordered layers) or variable (disordered layers). For both 1D and 2D ordered layers, we propose a geometrical scaling rule for the hydraulic permeability as a function of cylinder radius and solid volume fraction (porosity), which is a modification of a scaling rule previously reported by Clague and co-workers. The proposed modification is based on theoretical considerations and leads to significantly improved correspondence with simulation results. The hydraulic permeability of unstructured layers is found to be higher than that of structured layers of equal porosity for both 1D and 2D arrays. We propose a single parameter that can be easily determined experimentally to characterize the degree of disorder, as well as a generally valid correction factor in the proposed geometrical scaling rule to account for the influence of disorder on the hydraulic permeability.
\end{abstract}

DOI: 10.1103/PhysRevE.74.036301

PACS number(s): 47.56.+r, 47.11.-j, 47.15.G-

\section{INTRODUCTION}

Porous media play an important role in many natural and technological processes, e.g., ground water flow, biological transport phenomena, filtration and separation technologies, and production of textiles and paper. Prediction of the hydraulic permeability, which is a measure of the fluid conductance, is a well-known problem and was the subject of many studies. Due to its relevance, the problem was studied by numerous authors both analytically, numerically, and experimentally. A specific category of porous media is formed by fibrous media. The simplest model representation of a fibrous media is a one-dimensional (1D) or 2D array of solid cylinders. This is the topic of the present paper.

In the limit of the creeping flow (i.e., with Reynolds numbers based on the cylinder diameter $\ll 1$ ), predictions of the permeability $k$ of fibrous media date back to the work of Kuwabara [1,2] and Happel [3] in the late 1950s. Kuwabara reported results for rather low solid volume fractions $(\phi$ $<0.1$ ). Happel used a free-surface model to calculate the resistance to the flow for cylindrical objects. In the early 1980s, Sangani and Acrivos [4,5] performed numerical studies of the hydraulic permeability of periodic (square and hexagonal) arrays of cylinders with axes perpendicular to the flow direction for the full range of solid volume fractions $\left(0<\phi<\phi_{\mathrm{MAX}}\right)$. They proposed analytical correlations for the permeability $k=f(\phi)$ in the low and high limit of the solid volume fraction. Subsequently, Sangani and Yao [6] obtained numerical solutions of the permeability of randomly distributed arrays of cylinders with axes perpendicular to the flow direction. Due to the limitations of the computational resources, at the time, they did not perform systematic studies for varying random distributions at fixed solid volume fraction. The number of cylinders in the array was between 9

\footnotetext{
*Electronic address: Michal.Sobera@shell.com

†Electronic address: C.R.Kleijn@TUDelft.NL
}

and 16, depending on $\phi$. The enormous growth of the computational power since then facilitated simulations with a significantly higher number of cylindrical fibers. In recent years, several authors reported results for total array sizes between 100 and 1000 cylinders [7-9]. The focus in most recent studies is on $3 \mathrm{D}$ disordered media. Applying a singularity method [10], Clague and Philips [11] computed the permeability of ordered periodic arrays of cylinders and of disordered cubic (periodic) cells of cylindrical fibers. For the ordered case, their results exhibit good agreement to the data of Sangani and Acrivos [5] for $\phi<0.4$. In a subsequent paper, Clague et al. [12] used the lattice-Boltzmann technique to study similar geometries and extended their studies to include also bounded structures. They proposed that $k(\phi)$ scales with the square of the half distance $\delta$ between two adjacent cylinders. Manwart and Hilfer [13] proposed a method of reconstruction of random porous media by means of Monte Carlo simulations and reported results obtained from fluid flow simulations through the reconstructed media by means of lattice-Boltzmann and finite difference methods $[14,15]$.

From the mentioned studies it has become clear that, in addition to the porosity, the precise geometric configuration and (non)uniformity of the system are the dominant factors that influence its hydraulic permeability. A more detailed discussion will be provided in the subsequent sections.

For low Reynolds number ( $\mathrm{Re}$ ) flows, as discussed above, the pressure gradient over a porous medium is proportional to the fluid velocity (Darcy's law). For higher Re, the effect of fluid inertia becomes dominant. The theoretical and experimental study of the pressure drop (permeability) in disordered porous media at higher Re dates back to the work of Ergun [16] in the early 1950s, who proposed the well-known correlation for dimensionless drag $F^{*}=f(\phi, \mathrm{Re})$. Numerical simulations supported the understanding of the inertial effect, with a significant contribution of Andrade et al. [17], who simulated fluid flow in a disordered array of arbitrarily shaped fibers with axes perpendicular to the flow direction in 

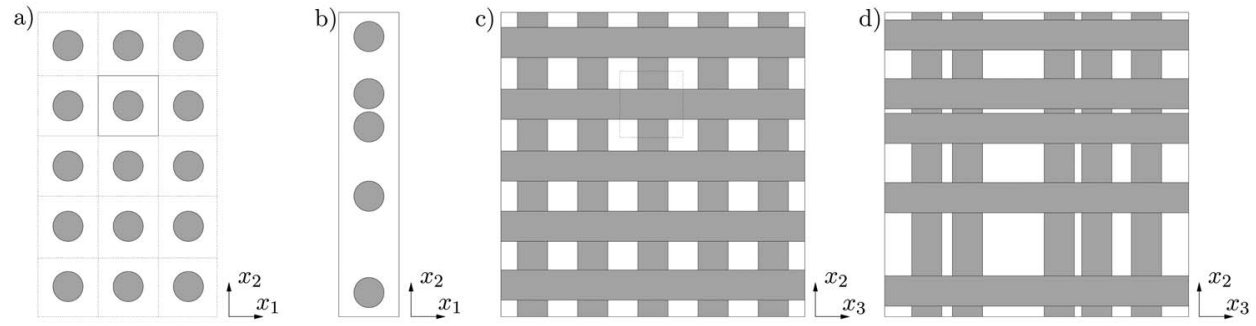

FIG. 1. Pictorial representation of the types of media studied in this paper: (a) periodic array of parallel cylinders with axes perpendicular to the flow direction, (b) disordered single-layer array of cylinders with axes perpendicular to the flow direction, (c) single-layer periodic array of perpendicularly crossing cylinders with axes perpendicular to the flow direction, and (d) single-layer disordered array of perpendicularly crossing cylinders with axes perpendicular to the flow direction. In all cases, the flow is in the $x_{1}$ direction.

order to investigate the origin of deviation from Darcy's law. The transition from linear to nonlinear dependence of the pressure drop on Re as stated by the Ergun correlation, was found to be characterized by a spatial distribution of the kinetic energy. In Ref. [18], Andrade et al. reported the results on the role of stagnant zones of randomly distributed arrays of arbitrarily shaped fibers by direct numerical solution of the Navier-Stokes equations. Recently, Papathanasiou et al. [19] made numerical evaluations of the Ergun [16] equation for square and hexagonal arrays of cylinders at solid volume fractions $0.3<\phi<0.6$ and $\operatorname{Re}$ between 0 and 160, while varying a geometrical parameter. In a subsequent paper, Markicevic and Papathanasiou [20] proposed and validated a model for the apparent permeability of nonuniform fibrous media for the same set of parameters as presented in Ref. [19]. Hill et al. [21,22] focused on the effect of fluid inertia at low and moderate Re in ordered and disordered arrays of spheres. The simulations were performed by means of the lattice-Boltzmann technique on a total number of spheres between 16 and 64 .

From the above discussion it is clear that many important aspects of fluid flow through (fibrous) porous media have been studied and that many important relationships have been revealed. Together with the Ergun modification for higher Re, Darcy's law gives a comprehensive description for the pressure drop as a function of permeability and fluid velocity. Several models for the permeability as a function of the solid volume fraction were proposed for certain classes of porous media. Clague et al. [12] proposed a simple scaling estimate $k / r_{\mathrm{f}}^{2}=f(\phi)$, for the influence of the cylinder radius on permeability at given porosity, which gives good qualitative agreement to experimental and simulation data. Several authors reported an increased permeability in systems consisting of randomly distributed cylinders, compared to ordered structures with the same porosity, but to this end no comprehensive studies were performed relating permeability to porosity, geometric factors, and distribution randomness.

In the present paper, we present such a systematic study by means of direct solution of the Navier-Stokes equations for low Re number flow through (i) single-layer arrays of parallel cylinders and (ii) single-layers of perpendicularly crossing cylinders, in both cases with the cylinder axes perpendicular to the flow direction. We aim to describe the hydraulic permeability as a function of the solid volume frac- tion (or porosity), the cylinder radius, and a single dimensionless disorder measure. The flow simulations were carried out by means of the finite volume based NavierStokes solver Fluent [23].

The work is split into three parts: In the first part (described in Secs. III A and IV A and intended to validate our numerical method), 2D simulations have been performed of the flow through periodic (square) arrays of parallel cylinders over the entire regime of possible solid fractions $\phi$ [Fig. 1(a)]. We compared our results to those reported in Refs. $[4,12]$. We also propose a modification of Clague's correlation for the dimensionless permeability $k / r_{\mathrm{f}}^{2}=f(\phi)$, leading to better quantitative agreement at high values of $\phi$. This correlation was found to be valid also for evenly distributed single layers of parallel cylinders.

In the second part (described in Secs. III B and IV B), 2D simulations have been performed of the flow through disordered single layers of parallel cylinders [Fig. 1(b)]. The goal of these simulations was to investigate the influence of the nonuniformity of the cylinder distribution on the permeability at fixed solid volume fraction. To this end, large numbers of nonuniformly distributed geometrical configurations were randomly generated and characterized by a single dimensionless disorder measure $\alpha$. We propose a simple but accurate correlation $\left[k / r_{\mathrm{f}}^{2}=f(\phi, \alpha)\right]$ between the dimensionless hydraulic permeability, the solid volume fraction, and this measure for the nonuniform distribution.

In the third part (described in Secs. III C, IV C, III D, and IV D), the proposed correlation was subsequently evaluated for various ordered [Fig. 1(c)] and disordered [Fig. 1(d)] single layers of perpendicularly crossing cylinders.

\section{BACKGROUND}

\section{A. Geometrical scaling estimate}

The hydraulic permeability is a measure of the fluid conductance of a porous material and is defined by Darcy's law [24]:

$$
\nabla P=\frac{\mu\langle\mathbf{v}\rangle}{\mathbf{K}},
$$

where $\mathbf{K}$ is the permeability tensor, $\langle\mathbf{v}\rangle$ is the superficial (averaged) velocity, $\boldsymbol{\nabla} P$ is the mean pressure gradient over the porous material, and $\mu$ is the fluid viscosity. In the 
present study, the focus is on the transverse component of the permeability (in the $x_{1}$ direction), which means that $\mathbf{K}=k_{11}$ $=k$.

To deduce a scaling rule for $k$ at low $\mathrm{Re}$ in periodic arrays of cylinders with their axes perpendicular to the flow direction [Fig. 1(a)], Clague et al. [12] performed a simple analysis of the Stokes flow equations, given by

$$
\begin{gathered}
\boldsymbol{\nabla} \cdot \mathbf{v}=0, \\
\mu \nabla^{2} \mathbf{v}-\nabla P=0 .
\end{gathered}
$$

Taking the half distance $\delta$ between two adjacent cylinders as the characteristic length scale over which rapid changes of the velocity occur in both the $x_{1}$ and $x_{2}$ directions, and taking $\langle v\rangle$ as the characteristic velocity, they deduced the following scaling estimate for Stokes flow:

$$
\nabla P \sim \mu \frac{\langle\mathbf{v}\rangle}{\delta^{2}} .
$$

By incorporating Darcy's law (1) into the last expression, a relation between permeability and mutual half distance between the cylinders is found:

$$
k \sim \delta^{2} \quad \text { or } \quad k=c \delta^{2} .
$$

For the configuration of Fig. 1(a) this leads to

$$
k=c r_{\mathrm{f}}^{2}\left(\frac{1}{2} \sqrt{\frac{\pi}{\phi}}-1\right)^{2},
$$

with $r_{\mathrm{f}}$ the cylinder radius (see Sec. III A). This is a first attempt to correlate the permeability to a geometrical factor other than the solid volume fraction. It gives a reasonably good prediction of the permeability in the dilute (low $\phi$ ) regime, but shows rather poor agreement in the dense (high $\phi)$ regime. We believe that this is a consequence of choosing the superficial fluid velocity $\langle v\rangle$ as velocity scale. We found it more appropriate to use the actual velocity $v=\langle v\rangle / \epsilon$ as the characteristic velocity scale. Here, $\epsilon$ is a ratio between open and total area (open frontal area fraction). This leads to a scaling estimate

$$
k=c \delta^{2} \epsilon .
$$

In the subsequent sections we will show that this modified scaling estimate gives a very good prediction of the permeability, also for high solid volume fractions $\phi$.

\section{B. Numerical method}

In the present work we numerically solved the steadystate Navier-Stokes flow equations by means of the unstructured, finite volume based flow solver Fluent v.6.1 [23]. In all cases studied, the Reynolds number based on the cylinder diameter was below 0.01 , effectively reducing the NavierStokes equations to the Stokes equations (2) and (3). Even if a code such as Fluent is probably not the most efficient way to solve the Stokes equations, we chose to use such a code because of the ease of generating the complex (especially 3D) geometries that we wanted to study. In all cases studied, the required computer resources were quite modest. In contrast to lattice-Boltzmann simulations reported in many previous studies, we used nonequidistantly discretized computational domains. Simulations of 2D square arrays of cylinders were performed using structured, body fitted meshes for $\phi<0.6$ and quadrilateral unstructured meshes for $\phi>0.6$. For disordered single layers of cylinders, we used triangular unstructured meshes. The 2D mesh sizes varied between 40 and $800 \mathrm{~K}$ grid cells. 3D simulations of singlelayer arrays of crossing cylinders were performed using unstructured tetrahedral meshes with $1 \mathrm{M}-4 \mathrm{M}$ grid cells. All meshes were made by use of the Gambit preprocessor [25]. To reduce the total number of cells, we applied local mesh refinement in the vicinity of the cylinders. Grid independence of the simulation results was tested by increasing the number of grid cells by a factor between 2 and 4 for a number of representative simulations.

To solve the governing equations, we employed a second order central difference spatial discretization scheme and to execute the pressure-velocity coupling we used the SIMPLE algorithm [26,27]. In all cases, we computed the velocity field for given geometry at fixed pressure gradient driving the flow. For the single-layer configurations, the inflow and outflow were located at a distance $5\left(2 r_{\mathrm{f}}+2\langle\delta\rangle\right)$ upstream and downstream from the layer. From the simulated velocity field, the average superficial velocity $\langle v\rangle$ was computed and subsequently the dimensionless permeability was calculated from Eq. (1) as

$$
k^{*}=\frac{k}{r_{\mathrm{f}}^{2}}=\frac{\mu\langle v\rangle \Delta x}{\Delta P} \frac{1}{r_{\mathrm{f}}^{2}} .
$$

\section{PROBLEM FORMULATION}

\section{A. Periodic square arrays and ordered single-layer arrays of parallel cylinders}

A periodic square array of parallel cylinders [Fig. 1(a)] is the simplest model representation of a fibrous medium. We used this configuration, which was studied extensively by Sangani and Acrivos [4,5] and Clague et al. [12], to perform a test of the applied numerical method. The pressure drop over a single layer in such a periodic array cannot be expected to be exactly equal to the pressure drop over a freestanding single layer of equidistant parallel cylinders, even for very low Reynolds numbers. The flow disturbance due to a single cylinder has a log dependence on the distance from the cylinder. In a periodic array, unlike in a single layer, this nondecaying velocity field is altered when the fluid reaches the next layer of fibers at which a force may be exerted. However, as will be shown in Sec. IV A, the differences between the pressure drop over a single layer in a periodic array and over a free standing single layer were negligibly small. A schematic representation of the unit computational cell for the periodic square array is shown in Fig. 2. The solid volume fraction is given by

$$
\phi=\frac{\pi r_{\mathrm{f}}^{2}}{\left(2 r_{\mathrm{f}}+2 \delta\right)^{2}} .
$$

The half distance between two cylinders in the periodic array can be written as 


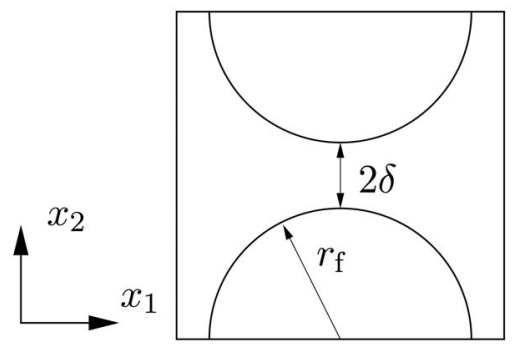

FIG. 2. Schematic representation of a $2 \mathrm{D}$ computational unit cell for a square periodic array of uniformly distributed cylinders. The flow is in the $x_{1}$ direction.

$$
\delta=r_{\mathrm{f}}\left(\frac{1}{2} \sqrt{\frac{\pi}{\phi}}-1\right)
$$

and the open frontal area fraction as

$$
\epsilon=\frac{\delta}{\delta+r_{\mathrm{f}}}
$$

The above is used as an input in Eq. (7) to compute the hydraulic permeability. It should be noted that the maximum solid volume fraction for a periodic array is $\phi=\pi / 4$.

\section{B. Disordered single-layer arrays of parallel cylinders}

A single-layer array of nonequidistantly distributed cylinders [Fig. 1(b)] is a slightly more complicated configuration which more closely resembles realistic, usually randomly distributed, single-layer porous media, such as woven and nonwoven filters, textiles, etc. A schematic description of a model geometry, with a unit cell consisting of (in this example case) $n=4$ solid cylinders of equal radius $r_{\mathrm{f}}$ is presented in Fig. 3. Within this part of the work we performed a systematic study on the influence of the randomness of the cylinder distribution (i.e., the variation in the half-distance $\delta$ between the cylinders) on the permeability of this simplified geometry, at given $r_{\mathrm{f}}$ and average $\langle\delta\rangle$.

We define the averaged solid volume fraction of an $n$-cylinder structure, as given in Fig. 3, as

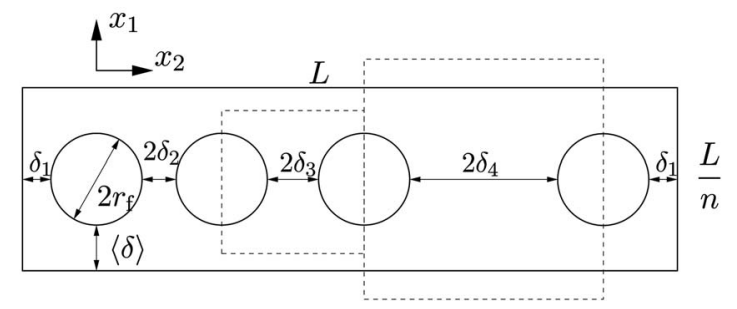

FIG. 3. A 2D periodic unit cell for a disordered single-layer array of parallel cylinders. The unit cell consists of $n=4$ cylinders and is defined by a mean volume fraction $\langle\phi\rangle$ and consequently a mean mutual cylinder distance $\langle\delta\rangle$ and a dimensionless disorder measure $\alpha$. The flow is in the $x_{1}$ direction.

$$
\left\langle\phi_{n}\right\rangle=\frac{n^{2} \pi r_{\mathrm{f}}^{2}}{L^{2}}, \quad \text { where } L=2\left(n r_{\mathrm{f}}+\sum_{i=1}^{n} \delta_{i}\right) \text {. }
$$

The sum of mutual cylinder-to-cylinder half distances as a function of mean volume fraction is given by

$$
\sum_{i=1}^{n} \delta_{i}=n r_{\mathrm{f}}\left(\frac{1}{2} \sqrt{\frac{\pi}{\left\langle\phi_{n}\right\rangle}}-1\right) .
$$

For a given mean solid volume fraction $\left\langle\phi_{n}\right\rangle$ and a given cylinder radius $r_{\mathrm{f}}$, many different random realizations of the cylinder distribution are possible, each of which will, expectedly, have a somewhat different permeability. Each random realization can be characterized by the probability distribution of the cylinder half distances $\delta_{i}$. We tried to characterize each random realization by a single, simplified dimensionless measure, based on the first (mean value $\langle\delta\rangle$ ) and the second (standard deviation $\sigma$ ) statistical moments of the probability distribution:

$$
\alpha=\frac{\sigma}{\left\langle\delta_{n}\right\rangle} .
$$

For single-layer arrays of cylinders the value of $\alpha$ can easily be determined experimentally, e.g. from inter-fiber distance measurements in microscope of the layer.

For equidistantly distributed cylinders $\alpha=0$. It can easily be shown that the maximum value of $\alpha$ depends on the number of cylinders in (a unit cell of) the system only: The standard deviation $\sigma$ and the dimensionless disorder measure $\alpha$ reach their maximum values when only one of the mutual distances has a nonzero value (e.g., $\delta_{1} \neq 0$ and $\left.\delta_{2,3,4 . . n}=0\right)$ ). In this case, $\delta_{1}$ at given $\left\langle\phi_{n}\right\rangle$ can be expressed as

$$
\delta_{1}=n r_{\mathrm{f}}\left(\frac{1}{2} \sqrt{\frac{\pi}{\left\langle\phi_{n}\right\rangle}}-1\right),
$$

and the standard deviation is given by

$$
\sigma_{\mathrm{MAX}}=\sqrt{\frac{\delta_{\mathrm{MAX}}^{2}-\frac{\delta_{\mathrm{MAX}}^{2}}{n}}{n}}=\frac{\delta_{\mathrm{MAX}}}{n} \sqrt{n-1},
$$

which leads to the expression for $\alpha_{\mathrm{MAX}}$

$$
\alpha_{\mathrm{MAX}}=\sqrt{n-1} .
$$

Note that, for a random placement of the cylinders (with restrictions to prevent overlap) within the domain, $\alpha \rightarrow 1$ for $n \rightarrow \infty$. This is a more realistic upper bound for $\alpha$ in technologically manufactured single-layer porous materials.

\section{Ordered single-layer arrays of perpendicularly crossing cylinders}

The simplest representation of a woven textile structure is a single-layer biperiodic array of perpendicularly crossing cylinders, which schematically is presented in Figs. 1(c) and 4. We define the solid volume fraction for such a defined structure by 


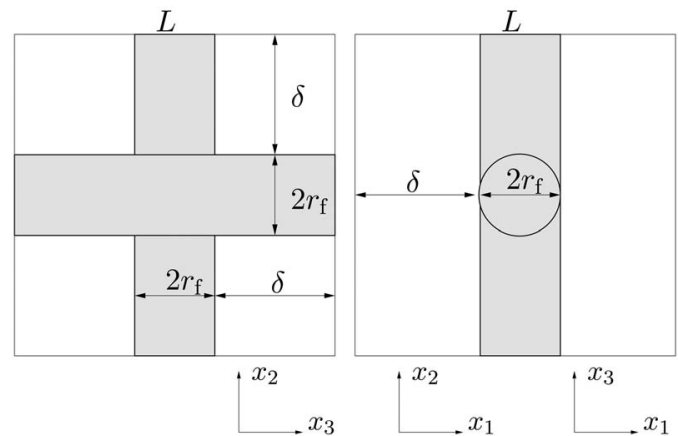

FIG. 4. A 3D biperiodic simulation unit cell for a single-layer periodic array of perpendicularly crossing cylinders. The fluid flows in the $x_{1}$ direction.

$$
\phi=\frac{2 \pi r_{\mathrm{f}}^{2} L-\frac{16}{3} r_{\mathrm{f}}^{3}}{L^{3}}=\frac{\frac{\pi}{2} r_{\mathrm{f}}^{2}\left(r_{\mathrm{f}}+\delta\right)-\frac{2}{3} r_{\mathrm{f}}^{3}}{\left(r_{\mathrm{f}}+\delta\right)^{3}} .
$$

By solving the above equation, we can express the mutual half distance $\delta$ between adjacent cylinders by

$$
\delta=\operatorname{Re}\left(A(\phi)+\frac{\pi r_{\mathrm{f}}^{2}}{6 \phi A(\phi)}-r_{\mathrm{f}}\right),
$$

where Re denotes the real part and:

$$
A(\phi)=\left[\frac{r_{\mathrm{f}}^{3}}{3 \phi}\left(\frac{\sqrt{6}}{12} \sqrt{\frac{-\pi^{3}+24 \phi}{\phi}}-1\right)\right]^{1 / 3} .
$$

\section{Disordered single-layer arrays of perpendicularly crossing cylinders}

Finally, we studied single-layer arrays of randomly distributed perpendicularly crossing cylinders [Fig. 1(d)]. A schematic representation of such a media, with a unit cell consisting (in this example) of $n=4$ cylinders, is presented in Fig. 5. In the present study, only structures with a square (in the $x_{2} x_{3}$ direction) unit cell were considered. Practically, this

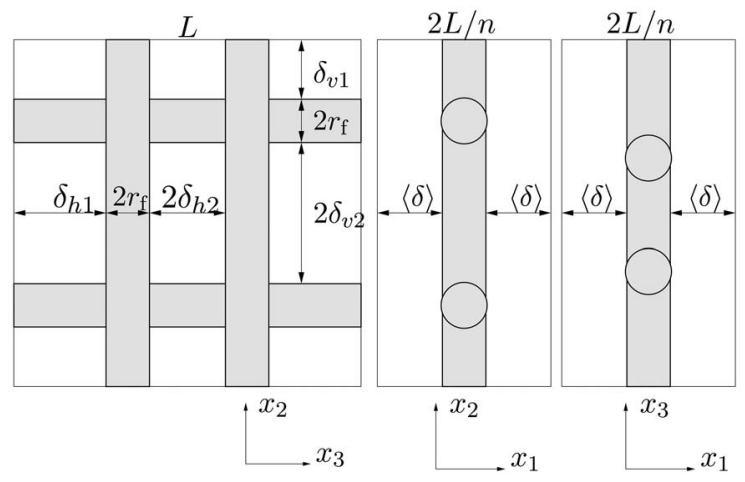

FIG. 5. A 3D biperiodic unit cell for a disordered single-layer array of perpendicularly crossing cylinders. This simulation cell consists of $n=4$ cylinders and is characterized by a mean solid volume fraction $\langle\phi\rangle$ and dimensionless disorder measure $\alpha$. The fluid flows in the $x_{1}$ direction. means that, although disorder and anisotropy is introduced at the small scale of the unit cell, the structures studied were ordered and isotropic at large, super-unit-cell, scales. This is an important difference with the disordered single-layer arrays of parallel cylinders described above, which are nonisotropic at all scales.

We define the averaged solid volume fraction of an $n$-cylinders structure, defined as in Fig. 5, by

$$
\left\langle\phi_{n}\right\rangle=\frac{n^{2}\left(\pi r_{\mathrm{f}}^{2} L-\frac{16}{3} r_{\mathrm{f}}^{3}\right)}{2 L^{3}},
$$

where

$$
L=n r_{\mathrm{f}}+2 \sum_{i=1}^{n / 2} \delta_{i}
$$

The average cylinder-to-cylinder distance is again given by Eq. (19).

In a similar way as for the disordered single-layer array of parallel cylinders, we introduce a nonuniformity measure $\alpha$, which can now be defined based on the hydraulic diameter:

$$
d_{\mathrm{H}}=\frac{4 \delta_{h} \delta_{v}}{2 \delta_{h}+2 \delta_{v}},
$$

where $\delta_{h}$ and $\delta_{v}$ are rectangle sizes in the $x_{3}$ and $x_{2}$ direction, respectively (see Fig. 5). The nonuniformity measure is given by

$$
\alpha=\frac{\sigma}{\langle\delta\rangle}=\sqrt{\frac{\sum_{i=1}^{n}\left(\frac{1}{2} d_{\mathrm{H} i}-\langle\delta\rangle\right)^{2}}{n\langle\delta\rangle^{2}}} .
$$

For the $\delta_{h} \gg \delta_{v}$, the structure approaches that of Sec. III B (Fig. 3). In this case, $\delta_{h}=2 \delta_{v}$ and the definition of $\alpha$ in Eq. (24) is identical to that of $\alpha$ in Eq. (14). For a system with uniformly distributed cylinders $\alpha=0$ and its maximal value depends on the number of cylinders in the unit cell and is given by Eq. (17).

\section{RESULTS AND DISCUSSION}

\section{A. Periodic square arrays and ordered single-layer arrays of parallel cylinders}

The hydraulic permeability, scaled with the square of the cylinder radius, calculated for a periodic array of parallel cylinders as a function of solid volume fraction is presented in Fig. 6. The results of our present simulations are compared to the numerical results of Sangani and Acrivos [4]. Additionally, we included the two analytical expressions as proposed by Sangani and Acrivos [4]. The first is valid in the low solid volume fraction regime:

$$
k^{*}=\frac{k}{r_{\mathrm{f}}^{2}}=\frac{-\frac{1}{2} \ln \phi-0.738+\phi-0.887 \phi^{2}+2.038 \phi^{3}}{4 \phi},
$$

and the second describes the dense regime 


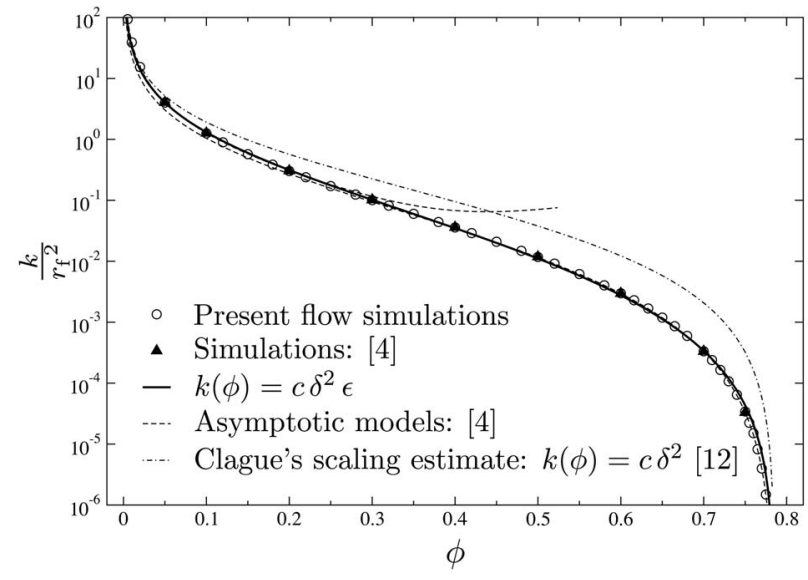

FIG. 6. Dimensionless hydraulic permeability $k / r_{\mathrm{f}}^{2}$ as a function of the solid volume fraction $\phi$ for a $2 \mathrm{D}$ square array of cylinders. Numerically obtained permeabilities, by means of the finite volume method, are compared to the results of Sangani and Acrivos (Ref. [4]) and to asymptotic models proposed by them. The results obtained by our modification of Clague's scaling estimate [Eq. (7)] are compared with the numerical and literature data and to the original form [Eq. (5)].

$$
k^{*}=\frac{k}{r_{\mathrm{f}}^{2}}=\frac{2 \sqrt{2}}{9 \phi}\left(1-\sqrt{\frac{4 \phi}{\pi}}\right)^{5 / 2} .
$$

The results of our present simulations match perfectly to the data of Sangani and Acrivos, as well as to the above analytical asymptotic expressions. This shows that the numerical method we employed, based on the finite volume flow simulations with local grid refinement near the cylinders, is suitable for this type of problem and leads to quantitatively accurate results.

We also computed the pressure drop over a free-standing single layer of parallel cylinders. It should be noted here, that for these flow simulations, the computational domain extended from $x_{1} \sim-5 \delta$ to $x_{1} \sim+5 \delta$, and the pressure drop was evaluated between the planes at $x_{1}=-(\delta+r)$ and $x_{1}=\delta+r$, rather than at $x_{1}=-\infty$ and $x_{1}=+\infty$ [see Fig. 1(b)]. Because of the nondecaying flow disturbance caused by a single layer of cylinders, differences can be expected between pressure drops for free-standing single layers evaluated over various up and downstream distances. These differences, however, were found to be small, i.e., less than $10 \%$ difference between the $\Delta x=10 \delta$ and $\Delta x=2 \delta$ cases. The difference between the pressure drop for a free-standing single layer evaluated over a distance $2 \delta$ (which is equal to the interlayer distance in a periodic array) and the pressure drop over a single layer in the periodic array was found to be less than $2 \%$ for $\phi>0.001$. These findings are in agreement with those of Sangani and Behl [28]. Such differences are extremely small when compared to the more than eight decades of variation in permeability covered by the data and correlation in Fig. 6. Therefore, we may conclude that the above results are also, with a very high accuracy, valid for freestanding ordered single-layer arrays of parallel cylinders.

Subsequently (Fig. 6), we validated our proposed modification of Clague's scaling estimate, as described in Sec. II A.

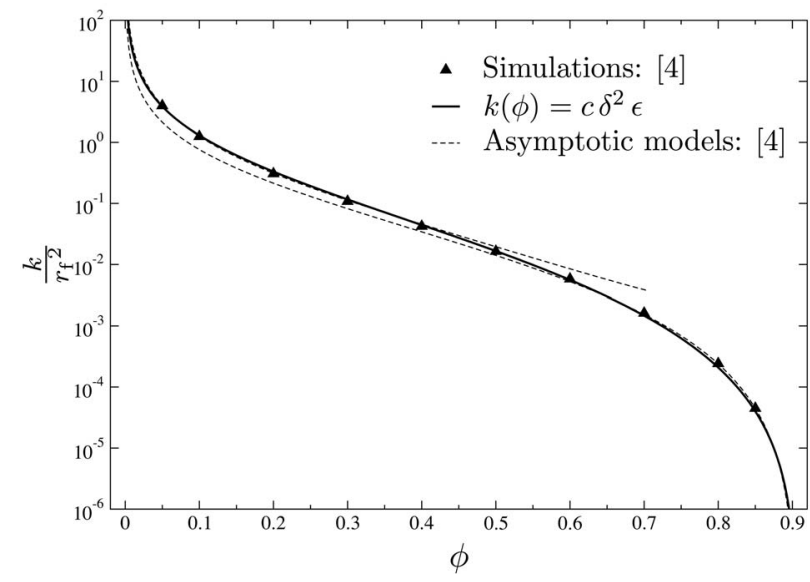

FIG. 7. Dimensionless hydraulic permeability $k / r_{\mathrm{f}}^{2}$ as a function of the solid volume fraction $\phi$ for a 2D hexagonal array of cylinders. Our model Eq. (7) is validated against the numerical literature data of Sangani and Acrivos (Ref. [4]), as well as the asymptotic Eqs. (29) and (30).

By adjusting the constant $c=0.59$ to obtain a best fit to the numerical data, the proposed model [Eq. (7)] gives an excellent prediction of the hydraulic permeability in the entire regime of the solid volume fractions compared to values from literature and from our present simulations. In the dense (high $\phi$ ) region, discrepancies can be observed between the numerical simulation results (which match the theoretical asymptotic solution) and the proposed correlation of Eq. (7). These discrepancies, which reach an asymptotically high relative value for $\phi \rightarrow \phi_{\mathrm{MAX}}=\pi / 4$, are due to a change in the scaling behavior. At high volume fraction, the length scale over which rapid changes of the velocity occur is $\sqrt{r_{\mathrm{f}} \delta}$ rather than $r_{\mathrm{f}}$. Nevertheless, our proposed correlation represents a significant improvement to that of Clague for a large range of volume fractions, accurately (i.e., within a few percent) predicting many decades of variation in the permeability for $0<\phi<0.70$. For larger $\phi$, the difference between our correlation and the exact asymptotic solution of Eq. (26) increases from, e.g., $\sim 10 \%$ at $\phi=0.72$ to $\sim 50 \%$ at $\phi=0.76$.

To further validate our proposed correlation, we compared its predictions to results reported by Sangani and Acrivos for a hexagonal array of cylinders [4], which is a staggered version of the square array. In Fig. 7 results obtained by Eq. (7) are compared to their numerical data and to analytical asymptotic expressions proposed in Ref. [4]. The maximum solid volume fraction for a hexagonal array is given by

$$
\phi_{\mathrm{MAX}}=\frac{\pi}{2 \sqrt{3}},
$$

and the mutual cylinders distance by

$$
\delta=\sqrt{\frac{\pi}{2 \sqrt{3} \phi}}-1 .
$$

This leads to the following asymptotic expression for the dilute regime: 


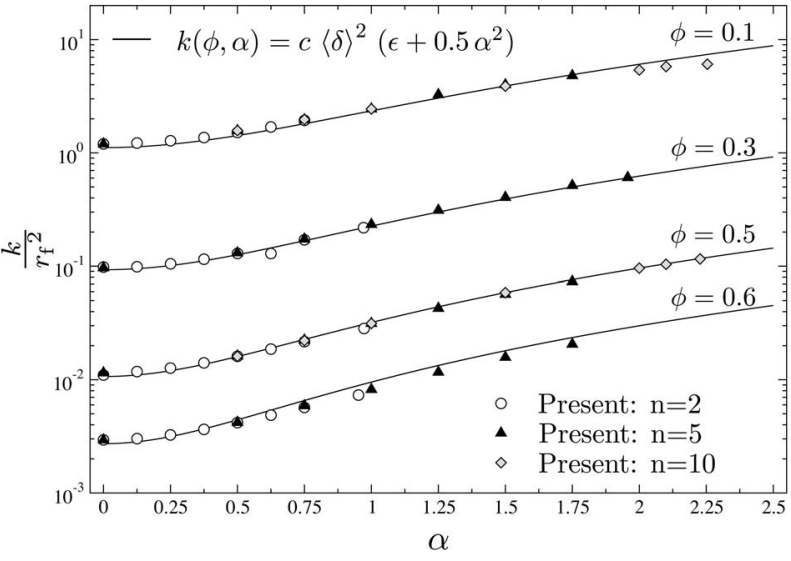

FIG. 8. Dimensionless hydraulic permeability $k / r_{\mathrm{f}}^{2}$ as a function of the dimensionless disorder measure $\alpha$ at four solid volume fractions $\phi$ computed for a disordered single-layer array of cylinders.

$$
k^{*}=\frac{k}{r_{\mathrm{f}}^{2}}=\frac{-\frac{1}{2} \ln \phi-0.745+\phi-0.25 \phi^{2}}{4 \phi},
$$

and for the dense regime

$$
k^{*}=\frac{k}{r_{\mathrm{f}}^{2}}=\frac{4 \sqrt{2}}{27 \phi}\left(1-\sqrt{\frac{2 \sqrt{3} \phi}{\pi}}\right)^{5 / 2},
$$

which should be compared to Eqs. (25) and (26).

Again, the constant $c$ from Eq. (7) was fitted to obtain the best fit to the numerical data of Sangani and Acrivos. With this $c=0.47$, the agreement of our proposed correlation Eq. (7), compared to the results of Sangani and Acrivos numerical flow simulations and to the analytical asymptotic expressions, is again very good for the full regime of solid volume fractions. As above, and for similar reasons, small discrepancies can be observed in the dense regime.

The above results show that our proposed modified correlation Eq. (7) of the dimensionless permeability $k / r_{\mathrm{f}}^{2}$ as a function of solid volume fraction $\phi$ gives excellent predictions for periodic arrays of cylinders, both square and hexagonal, as well as for ordered single layers of parallel cylinders. These configurations are the simplest representations of fibrous porous media. In the following section we will test the performance of the proposed correlation for slightly more complicated configurations.

\section{B. Disordered single-layer arrays of parallel cylinders}

Simulations were conducted for periodic systems with unit cells consisting of $n=2,5$, and 10 cylinders, each with four different solid volume fractions: $\phi=0.1,0.30 .5$, and 0.6. As we showed by Eq. (17), the maximum value of $\alpha$ depends only on the total number of cylinders in a unit cell of the system. We are searching for a relation between the scaled hydraulic permeability $k^{*}$ and the disorder measure $\alpha$ at each solid volume fraction.

Analyzing Fig. 8, we can observe that the hydraulic permeability increases with increased $\alpha$. It is remarkable thatfor the range of $\alpha$ 's common to all values of $n$ (i.e., $0<\alpha$
$<1)$ - the permeability is the same for systems with different $n$. This shows that the proposed approach of describing the measure of disorder in the medium via the single parameter $\alpha$ leads to very good and generic results.

After the preliminary qualitative study, we aimed to give a quantitative description of the results in Fig. 8. Considering the simplest disordered system, with $n=2$, we can assume that the total permeability is a linear superposition of two components. Following Eq. (7) this leads to

$$
k=\frac{k_{1}+k_{2}}{2}=\frac{1}{2}\left(c_{1} \delta_{1}^{2} \epsilon_{1}+c_{2} \delta_{2}^{2} \epsilon_{2}\right) .
$$

A two-cylinder system is characterized by a deterministic relation between $\delta_{1}$ and $\delta_{2}$ at given $\alpha$ :

$$
\delta_{1}=\langle\delta\rangle(1+\alpha) \text { and } \delta_{2}=\langle\delta\rangle(1-\alpha),
$$

where $\langle\delta\rangle$ is the average cylinder to cylinder distance. For simplification we can assume that $c_{1}=c_{2}=c$ and that the characteristic velocity is proportional to the average open frontal area fraction $\epsilon_{1}=\epsilon_{2}=\epsilon$, leading to

$$
k=c\langle\delta\rangle^{2}\left(1+\alpha^{2}\right) \epsilon .
$$

The results obtained with this model [Eq. (33)] match with numerical results over entire $\alpha$ domain for a low solid volume fractions. For higher $\phi$, however, the agreement is good only for small $\alpha$. This is due to the improper characteristic velocity choice based on the average open frontal area fraction.

In order to find a better model describing the hydraulic permeability of disordered systems, we considered Stokes flow in a two-cylinder unit cell. Assuming a constant pressure drop, we can write

$$
\nabla P \sim \mu \frac{v_{1}}{\delta_{1}}=\mu \frac{v_{2}}{\delta_{2}},
$$

and

$$
v_{1} \delta_{1}+v_{2} \delta_{2}=2 \frac{\langle v\rangle}{\epsilon}\langle\delta\rangle
$$

Combining Eqs. (34) and (35) with Darcy's law (1), we find that the hydraulic permiability $k$ is given by

$$
k=c\langle\delta\rangle^{2}\left(1+3 \alpha^{2}\right) \epsilon .
$$

However, also Eq. (36) does not perform well in reproducing our simulation results, and differences between this relation and the CFD simulations are again significant. The reason for the failure of both Eqs. (33) and (36) must be found in the underlying assumption that the flow and pressure fields around an $n=2$ nonequidistant unit cell can be obtained from a simple linear combination of the flow and pressure fields that would be present around its two constituent $n=1$ unit cells. This is untrue because of the complex interaction between the two flow and pressure fields, and because of the nonlinear relationships between pressure drop, velocity, and interfiber distance.

Without rigid theoretical explanation, we found the following empirical relation between $k, \alpha$, and $\epsilon$, which should be compared to Eqs. (33) and (36): 


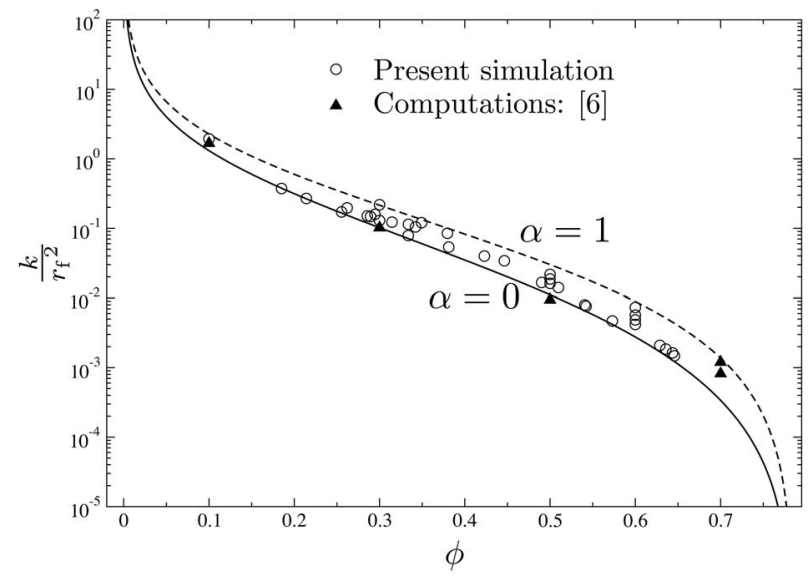

FIG. 9. Dimensionless hydraulic permeability $k / r_{\mathrm{f}}^{2}$ as a function of the solid volume fraction $\phi$. The results of our model at two values of disorder measure $\alpha$ are compared to the literature and our numerical data.

$$
k=c\langle\delta\rangle^{2}\left(0.5 \alpha^{2}+\epsilon\right) .
$$

This correlation is equal to Eq. (7), multiplied by a correction factor $\left(1+0.5 \alpha^{2} / \epsilon\right)$. This correction factor should be seen as an approximation for the unknown nonlinear relationship between the characteristic velocity and length scales in a disordered array, and $\langle v\rangle,\langle\delta\rangle, \epsilon$, and $\alpha$. For $\alpha=0$, i.e., an equidistant array, the correction factor equals 1 and Eq. (37) reduces to Eq. (7). Equation (37) is simple to use in practice and gives very good results over the entire range of $\alpha$ at each solid volume fraction that we studied, see Fig. 8 . The overall agreement with our numerical data is excellent. Again, small discrepancies can be observed in the dense regime.

In Fig. 9, the hydraulic permeability calculated with Eq. (37) for two different values of $\alpha$ as a function of solid volume fraction is presented. The solid line $(\alpha=0)$ represents a uniformly distributed porous structure, the dashed line ( $\alpha$ $=1$ ) corresponds to a randomly generated (Poisson distributed) system with a large total amount of cylinders $(n \rightarrow \infty)$. The area in between these two lines gives the most probable values of permeability for randomly distributed systems. Note that the permeability of disordered layers can be more than a factor of 3 higher than that of ordered layers, at identical solid volume fraction and cylinder radius. Also in Fig. 9 , we included results of our numerical simulations for a large number of randomly generated structures, with $n=2,5$, and 10 (white circles). Additionally, literature data from Sangani and Yao [26] for a 2D multilayer array of randomly distributed cylinders have been included. It should be noted that all of the numerically computed data, both from the present work and from the literature, lay in between the two curves, indicating that these indeed form an envelope of the most probable permeability of layers consisting of a large number of-randomly (Poisson) distributed parallel cylindrical fibers.

\section{Ordered single-layer arrays of perpendicularly crossing cylinders}

In Fig. 10 the hydraulic permeability as a function of solid volume fraction for a single-layer array of perpendicularly

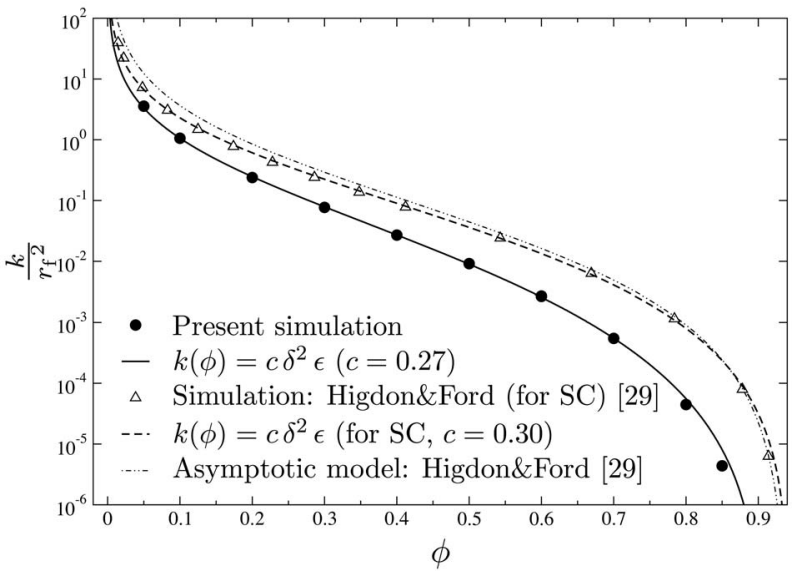

FIG. 10. Dimensionless hydraulic permeability $k / r_{\mathrm{f}}^{2}$ as a function of the solid volume fraction $\phi$ for an ordered single-layer array of perpendicularly crossing cylinders. Proposed model Eq. (7) is compared to the numerical results.

crossing cylinders is presented. The results of our numerical simulations are compared to the model [Eq. (7)]. For this case we used a relation for the mutual cylinders distance given by Eq. (19). Again, the results of numerical simulations correspond very well to the prediction of our modified Clague's scaling estimate. Some discrepancy is seen in the dense regime, again probably due to the same effect as described in Sec. IV A. By analysis of the Stokes flow equations, in a similar way as in Sec. II A, we can expect that the value of the constant $c$ should be approximately two times smaller for a single-layer array of perpendicularly crossing cylinders as for a single layer or square periodic array of parallel cylinders. By fitting to the numerical data, we found $c=0.27$ for a single-layer array of perpendicularly crossing cylinders, which is indeed 2.2 times smaller compared to the value $c=0.59$ obtained for the single layer and square periodic array of parallel cylinders. This shows that our proposed scaling estimate gives very good predictions of the hydraulic permeability of widely varying single-layer configurations.

Higdon and Ford [29] studied the hydraulic permeability of various three-dimensional structures built of cylindrical fibers. In Fig. 10 we compare their results for a single cubic lattice to the predictions from our model [Eq. (7)], using $c$ $=0.30$ to obtain a best fit to their numerical data. Thus, we found a very good agreement between our model and the calculations of Higdon and Ford. Again, some discrepancy with the numerical data and the asymptotic model can be observed in the dense region. Similar to the periodic array of cylinders, this discrepancy (reaching an asymptotically high relative value for $\phi \rightarrow \phi_{\mathrm{MAX}}$ ) is due to a changing scaling behavior at high values of $\phi$.

\section{Disordered single-layer arrays of perpendicularly crossing cylinders}

Finally, we focus on disordered single-layer arrays of perpendicularly crossing cylinders, limiting ourselves, as stated earlier, to structures with a square unit cell. The hydraulic permeability as a function of $\alpha$ [now defined based on the 


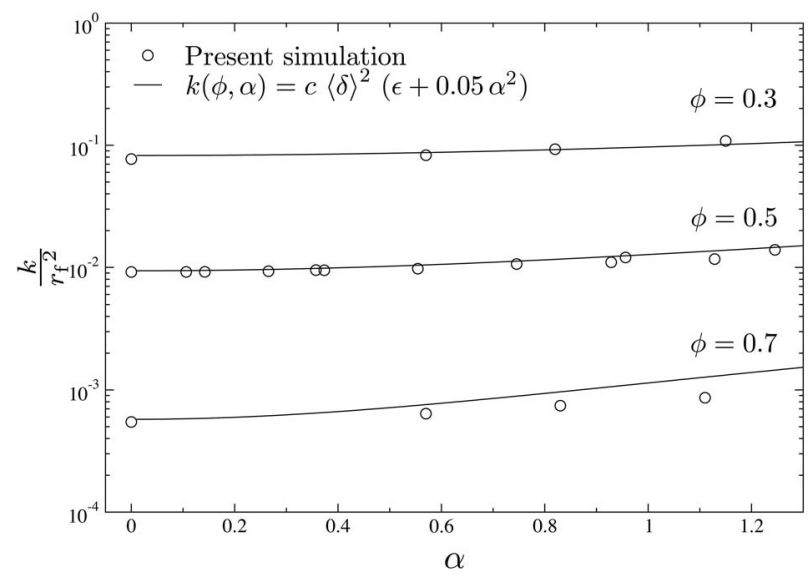

FIG. 11. Dimensionless hydraulic permeability $k / r_{\mathrm{f}}^{2}$ as a function of the dimensionless disorder measure $\alpha$ at three solid volume fractions $\phi$ computed for a 3D disordered single-layer array of perpendicularly crossing cylinders.

variation of the hydraulic diameter according to Eq. (24)] is presented in Fig. 11. In this graph numerical data computed at varied $\phi$ and $\alpha$ are presented. Assuming a similar relationship as found for disordered single layers of parallel cylinders, we found the following empirical best fit to the numerical data:

$$
k=c\langle\delta\rangle^{2}\left(\epsilon+0.05 \alpha^{2}\right) .
$$

The agreement is satisfactory at low and moderate solid volume fraction. For dense systems (high $\phi$ ) the agreement is poor for $\alpha>0.5$.

From Fig. 11 we can clearly see that at each solid volume fraction the increase of the hydraulic permeability with increased $\alpha$ is significantly smaller than for the arrays of parallel cylinders. This must probably be ascribed to the largescale isotropy of the structures studied here, as opposed to the anisotropic nature of disordered single layers of parallel cylinders.

\section{CONCLUSIONS}

We studied the hydraulic permeability of ordered and disordered single layers of fibrous materials for low Reynolds number (based on the fiber diameter) flows. Our numerical method was extensively validated against data from literature and was found to accurately reproduce these data.

As models of ordered layers we studied one-dimensional arrays of parallel, equidistant cylinders, and two-dimensional arrays of equidistant, perpendicularly crossing cylinders, in both cases with axes perpendicular to the flow direction. The hydraulic permeability of such layers was found to scale quadratically with the cylinder-to-cylinder distance and linear with the open frontal area fraction. The quadratic dependence on the cylinder-to-cylinder distance was proposed earlier by Claque and co-workers. The additional linear dependence on the open frontal area fraction, proposed in the present paper, is an extension of that scaling rule. It is based on theoretical considerations and leads to significantly improved correspondence with observed permeabilities.

As models of disordered layers we studied onedimensional arrays of parallel, nonequidistant cylinders, and two-dimensional-square unit cell-arrays of perpendicularly crossing, nonequidistant cylinders, in both cases with axes perpendicular to the flow direction. A single parameter was proposed as a measure for the variation in the cylinderto-cylinder distance. This parameter is zero for ordered layers, and approaches 1 for randomly generated arrays with large numbers of cylinders. In agreement with observations in literature, the hydraulic permeability was found to increase (by up to an order of magnitude) with increasing disorder. For disordered layers of parallel cylinders, we proposed a simple extension of the above scaling rule for ordered layers. This scaling rule was found to lead to very accurate predictions for a large variety of (randomly generated) disordered layers, confirming the validity of the use of a single parameter in characterizing the disorder. A similar scaling rule was also found to apply for disordered-square unit cell-layers of perpendicularly crossing cylinders. However, for these layers the increase in permeability with increasing disorder was much less pronounced.

\section{ACKNOWLEDGMENTS}

Research on this project was supported and financed by the Prins Maurits Laboratory of the Netherlands Organization for Applied Scientific Research (TNO). The authors would like to thank Dr. Drona Kandhai for many useful discussions.
[1] S. Kuwabara, J. Phys. Soc. Jpn. 12, 291 (1957).

[2] S. Kuwabara, J. Phys. Soc. Jpn. 14, 527 (1959).

[3] J. Happel, AIChE J. 5, 174 (1959).

[4] A. Sangani and A. Acrivos, Int. J. Multiphase Flow 8, 193 (1982).

[5] A. Sangani and A. Acrivos, Int. J. Multiphase Flow 8, 343 (1982b).

[6] A. Sangani and C. Yao, Phys. Fluids 31, 2435 (1988).

[7] D. Koch and A. Ladd, J. Fluid Mech. 349, 31 (1997).

[8] G. Liu and K. Thompson, Int. J. Numer. Methods Fluids 38,
1009 (2002).

[9] R. Verberg and A. J. C. Ladd, Phys. Rev. E 60, 3366 (1999).

[10] T. Dabros, J. Fluid Mech. 156, 1 (1985).

[11] D. Clague and R. Phillips, Phys. Fluids 9, 1562 (1997).

[12] D. Clague, B. D. Kandhai, R. Zhang, and P. M. A. Sloot, Phys. Rev. E 61, 616 (2000).

[13] C. Manwart and R. Hilfer, Phys. Rev. E 59, 5596 (1999).

[14] C. Manwart, U. Aaltosalmi, A. Koponen, R. Hilfer, and J. Timonen, Phys. Rev. E 66, 016702 (2002).

[15] C. Manwart and R. Hilfer, Physica A 314, 706 (2002). 
[16] S. Ergun, Chem. Eng. Prog. 48, 89 (1952).

[17] J. S. Andrade, U. M. S. Costa, M. P. Almeida, H. A. Makse, and H. E. Stanley, Phys. Rev. Lett. 82, 5249 (1999).

[18] J. S. Andrade, M. P. Almeida, J. Mendes Filho, S. Havlin, B. Suki, and H. E. Stanley, Phys. Rev. Lett. 79, 3901 (1997).

[19] T. Papathanasiou, B. Markicevic, and E. Dendy, Phys. Fluids 13, 2795 (2001).

[20] B. Markicevic and T. Papathanasiou, Phys. Fluids 14, 3347 (2002).

[21] R. Hill, D. Koch, and A. Ladd, J. Fluid Mech. 448, 213 (2001).
[22] R. Hill, D. Koch, and A. Ladd, J. Fluid Mech. 448, 243 (2001).

[23] Fluent 6 User's Guide (Fluent Inc., Lebanon, USA, 2001).

[24] S. Whitaker, Transp. Porous Media 3, 1 (1986).

[25] Gambit 2 User's Guide (Fluent Inc., Lebanon, USA, 2001).

[26] J. Ferziger and M. Peric, Computational Methods for Fluid Dynamics (Springer, Berlin, Germany, 1996).

[27] S. Patankar, Numerical Heat Transfer and Fluid Flow (Hemisphere, New York, 1980).

[28] A. Sangani and S. Behl, Phys. Fluids A 1, 21 (1989).

[29] J. Higdon and G. Ford, J. Fluid Mech. 308, 341 (1996). 